\title{
Laser-Doppler-Vibrometer calibration by laser stimulation
}

\author{
H. Volkers ${ }^{1}$ and Th. Bruns ${ }^{2}$ \\ ${ }^{1}$ Physikalisch-Technische Bundesanstalt, Braunschweig und Berlin, Germany, henrik.volkers@ptb.de \\ ${ }^{2}$ Physikalisch-Technische Bundesanstalt, Braunschweig und Berlin, Germany, thomas . bruns@ptb.de
}

\begin{abstract}
A new set-up for primary laser vibrometer calibration was developed and tested at the acceleration laboratory of PTB. Contrary to existing set-ups, this configuration makes use of electro-optical excitation. While avoiding the limitations imposed by mechanical motion generators in classic set-ups, the new method still encompasses all components of commercial laser vibrometers in the calibration and thus goes beyond the current capabilities of the purely electrical excitation schemes.
\end{abstract}

Keywords: primary calibration, laser doppler vibrometer, LDV calibration

\section{INTRODUCTION}

Laser Doppler vibrometers (LDV) are great tools for all kind of vibration measurements, especially for the use as a primary reference for calibration of accelerometers as described in the standards [1, 2].

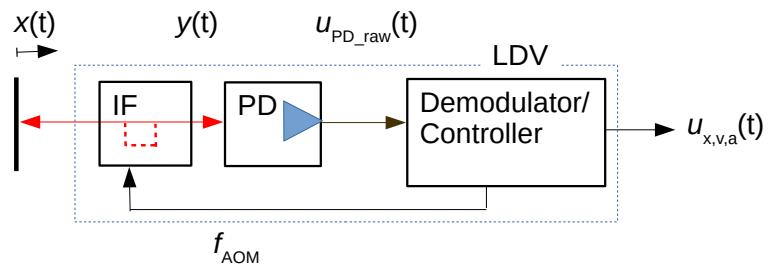

Figure 1: Schematic signal chain of a laser Doppler vibrometer

Figure 1 shows a schematic diagram of the signal flow in an LDV. At the measurement point, the motion quantity $x(t)$ is measured via a laser beam that passes an interferometer (IF) including an acoustooptical modulator (AOM) and finally illuminates a photodetector (PD) with an intensity modulated by interference according to

$$
I(t)=I_{0} \cdot \sin \left(2 \pi f_{\mathrm{AOM}} \cdot t+4 \pi \frac{x(t)}{\lambda}+\tau_{0}\right)+b+e_{\text {noise }}
$$

with $f_{\mathrm{AOM}}$ as the frequency of the AOM, $\lambda$ the wavelength of the laser, $\tau_{0}$ a constant delay due to the time of flight of the laser light, a constant bias intensity $b$ typical for interference and a noise component $e_{\text {noise }}$, e.g. from stray ambient light.

The voltage output $u_{\mathrm{PD}_{\text {raw }}}$ of the photo detector follows the intensity with a certain additional delay and additional noise from embedded amplifiers and feeds into the demodulation stage of the LDV controller. The internal processing of a commercial LDV is generally unknown to the user but probably follows an arctangents demodulation scheme as described in [3].

The demodulated analog (voltage) output of the instrument is then characterized by its complex transfer functions $S$ in the frequency domain:

$$
S_{\mathrm{ux}}(f)=\frac{U_{\mathrm{x}}(f)}{X(f)}
$$

where the signal delay is characterized by the phase of this complex quantity.

\section{EXISTING METHODS}

Existing calibration methods and set-ups either follow the classical approach of measuring a mechanical vibration [4, 5] or use an electrical excitation of the LDV controller and thus simulate the optical laser head.

For the former set-up a typical primary accelerometer calibration system is utilized where a reference LDV measures the motion of a shaker's armature to provide a reference acceleration signal. The measurement beam of the device under test (DUT) is coaligned to the reference beam using a beam splitter and points at the same spot on the armature surface. In the optimized set-up [6] only a single laser beam needs to be adjusted, hence, inaccuracies of the co-alignment are avoided.

This method provides a significant smaller uncertainty for LDV calibration than for the classical accelerometer calibration. However, it still suffers from the mechanical limitations of the utilized shaker system in terms of a limited frequency and amplitude range and non-ideal motion.

The second method substitutes the laser head of the DUT with an electrical signal generator. The signal generator provides the frequency modulated input to 


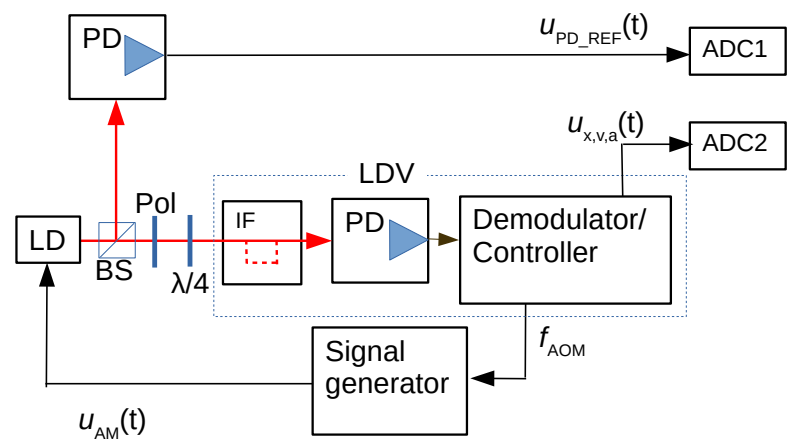

Figure 2: Schematic of a LDV calibration setup with amplitude-modulated laser diode stimulation

the LDV controller of the DUT, simulating the photo diode output of the laser head. By providing a corresponding FM signal, a very wide variety of virtual motion patterns can be simulated under almost ideal conditions. By simultaneous sampling of the generator signal and the DUT output a precise determination of the complex transfer function of the DUT is possible.

This method, however, requires good knowledge of the working principle of the hardware in order to provide adequate signal levels and carrier frequencies to the controller. In addition, it is not able to account for any signal preconditioning performed within the original laser head. On the other hand it suffers from far less limitations by avoiding any mechanical components[7].

\section{STIMULATION BY AMPLITUDE MOD- ULATED LASER SOURCE}

The basic idea of the new set-up evolves from the Eq. (1) and Figure 1 and is shown in Figure 2, The photo diode, being the central sensing part of the LDV, cannot distinguish the cause of an intensity variation. Whether it is the result of optical interference or simply is an intensity variation caused by a modulated external light source makes no difference. If an appropriate light source with a suitable amplitude modulation following Eq. (1) is targeted at its sensing element, the LDV response will be identical to the respective interference caused by real motion.

This approach combines the benefits of those existing set-ups described above, while avoiding the disadvantages. While ommiting any mechanical moving components, that may limit the scope or accuracy, it still includes the potential preprocessing of the laser head in the calibration. All requested excitations for the calibration of LDVs can be provided by electrooptical means.

In the new calibration set-up at PTB (c.f. Figure 2), the light source is a common $10 \mathrm{~mW}$ laser diode (LD) of a wavelength of $635 \mathrm{~nm}$, well matching the LDV's He-Ne wavelength. The bias current is adjusted such that the mean beam power entering the LDV is less than $1 \mathrm{~mW}$, matching approximately the typical output power of the LDV's laser.

An non-polarising beam splitter (BS) separates about $50 \%$ of the light which is directed to a reference photo diode (FEMTO HCA-S-400M) of a bandwidth of $400 \mathrm{MHz}$ and a known time delay [8]. An RF generator with phase modulation capability provides the modulation current for the laser diode. The modulation depth is adjusted in a range of $30 \%$ to $50 \%$ and monitored by the reference photo diode.

A polarization filter $(\mathrm{Pol})$ and a $\lambda / 4$ plate between LD and LDV ensure that circular polarized laser light enters the interferometer. The majority of the emitted light from the LD is already linear polarized, however, for the first set-up, the orientation of the polarity is unknown, hence, a polarization filter eases the initial orientation of the $\lambda / 4$ plate. Not shown in Figure 2 is the collimator lens of the laser diode and two aperture plates used to ease the alignment of the stimulating laser diode unit with the LDV's beam line.

\section{SIGNAL GENERATION AND PROCESS- ING}

The data acquisition system is based on a PXI system controlled by a LabVIEW program and provides two synchronized ADC channels for the acquisition of the reference signal and the DUT output. While the final stage of the set-up is supposed to include an arbitrary waveform generator and the ability to phase lock the generator or the whole PXI system to the LDV carrier frequency, the preliminary results given here were acquired by utilizing an RF generator (Type Agilent E4400B). The system is similar to the acquisition systems already used and validated for the primary acceleration calibration facilities at PTB.

The synchronous data acquisition was performed at $200 \mathrm{MS} / \mathrm{s}$ to account for the high carrier frequency. The demodulation of the reference followed the validated arctangents demodulation of PTB's national primary calibration standards. Finally, the evaluation of the simulated motion used the usual three-parameter sine-approximation.

\subsection{Determination of time delays}

Figure 3 shows the involved time delays of the calibration set-up depicted in Figure 2. All components except $H_{\mathrm{LDV}}$ are assumed to have a true time delay, i.e. the time delay is constant within the frequency ranges observed.

The time delay

$$
\tau_{\text {cor }}=\frac{\Phi_{\text {cor }}}{\omega}, \text { with } \omega=2 \pi f
$$




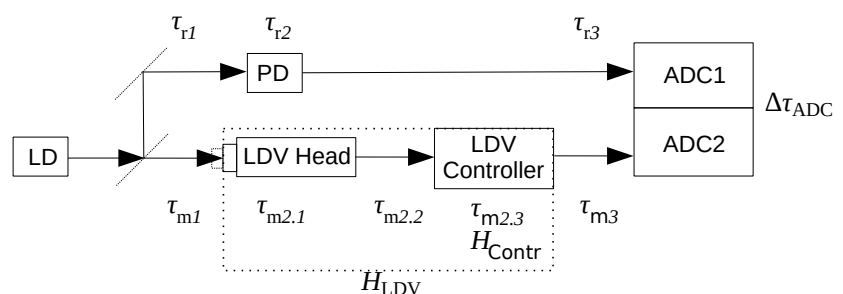

Figure 3: Time delays of the signal chains

to correct the measured transfer function $H_{\text {meas }}$ to get $H_{\mathrm{LDV}}$

$$
H_{\mathrm{LDV}}(\omega)=\left|H_{\text {meas }}(\omega)\right| \cdot e^{j \omega\left(\Phi_{\text {meas }}-\Phi_{\text {cor }}\right)}
$$

is calculated as

$$
\tau_{\mathrm{cor}}=\tau_{\mathrm{r} 1}-\tau_{\mathrm{m} 1}+\tau_{\mathrm{r} 2}+\tau_{\mathrm{r} 3}-\tau_{\mathrm{m} 3}+\Delta \tau_{\mathrm{ADC}}
$$

with the values:

$$
\begin{aligned}
\tau_{\mathrm{r} 1}-\tau_{\mathrm{m} 1} & =-545(5) \mathrm{mm} \cdot c_{\mathrm{air}}=-1.82(3) \mathrm{ns}(6) \\
c_{\mathrm{air}} & =2.9971 \times 10^{8} \mathrm{~m} / \mathrm{s}
\end{aligned}
$$

being the difference of the laser beam lengths measured from the reference surface of the LDV head stated in the manual. Its uncertainty covers the unknown delays of the $\lambda / 4$ plate and the polarization filter. The photo diode delay $\tau_{\mathrm{r} 2}$ was measured in [8] as

$$
\tau_{\mathrm{r} 2}=3.10(8) \mathrm{ns} .
$$

The time delay difference between the two simultaneously sampled channels, each with an RG58 cable of $2 \mathrm{~m}$ length, representing the term $\tau_{\mathrm{r} 3}-\tau_{\mathrm{m} 3}+\Delta \tau_{\mathrm{ADC}}$, is measured by feeding both cable ends with a common signal from the generator via a power splitter MiniCircuits ZFSC-2-4. By doing multiple measurements with swapped cables at the power splitter and ADC inputs we found

$$
\tau_{\mathrm{r} 3}-\tau_{\mathrm{m} 3}+\Delta \tau_{\mathrm{ADC}}=0.15(5) \mathrm{ns}
$$

including variances due to remounting. Putting the results into (5) gives

$$
\tau_{\text {cor }}=1.43(10) \mathrm{ns}
$$

The measurement of a time delay was performed in a two-step process. In the first step, a signal with a carrier frequency of $40 \mathrm{MHz}$ and an FM sine modulation of $500 \mathrm{~Hz}$ and a modulation depth of $3.15 \mathrm{MHz}$ was applied and a coarse time delay was determined by the maxima of a computed cross correlation, see Figure 4. In the final step the modulation was turned off and the phase difference of the $40 \mathrm{MHz}$ carrier signal was measured by applying a three parameter sine approximation. This process was chosen to be able to measure time delays of heterodyne signal outputs of LDV controllers with a time delay greater than one period of the carrier signal ( $25 \mathrm{~ns}$ in this case) due to internal signal processing like amplitude stabilisation or filter.
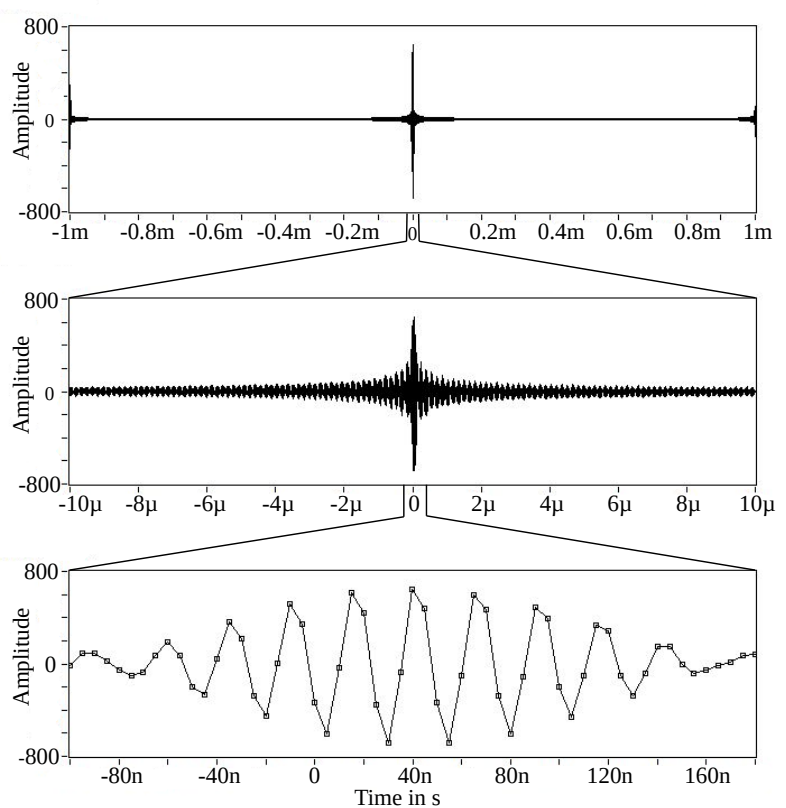

Figure 4: Cross correlation of the two ADC channels at a sample rate of $200 \mathrm{MHz}$, both fed with a $40 \mathrm{MHz}$ FM signal modulated with a $500 \mathrm{~Hz}$ sine of $3.15 \mathrm{MHz}$ modulation depth, on different time scales, with one of the two connection cables being about $8 \mathrm{~m}$ longer, resulting in a delay of 41.90(5)ns.

\section{RESULTS}

First tests with a LDV controller Polytec OFV5000-KU and a laser head OFV 353, connected via a $5 \mathrm{~m}$ cable, were performed and figure 5 shows a measured frequency response obtained with the new setup. The LDV is based on a carrier frequency $f_{\mathrm{AOM}}$ of $40 \mathrm{MHz}$.

The LDV controller settings were:

- Velocity decoder: VD-01

- Range: $1 \mathrm{~m} / \mathrm{s} / \mathrm{V}$

- Max. frequency: $50 \mathrm{kHz}$

- Tracking filter: off

- Low pass filter: $100 \mathrm{kHz}$

- High pass filter: off

The frequency range in terms of the simulated vibration was $100 \mathrm{~Hz}$ to $30 \mathrm{kHz}$ and an amplitude of $1 \mathrm{~m} / \mathrm{s}$ leading to a modulation depth of $3.159 \mathrm{MHz}$. The plots show the relative deviation in magnitude and the absolute phase of the analog velocity output in relation to the demodulated intensity stimulus on a linear frequency scale. The nearly linear phase corresponds to a delay of about $7.52 \mu \mathrm{s}$.

\section{OUTLOOK}

Instead of a photo diode with known delay, a known laser source would obviate the beam splitter and photo 


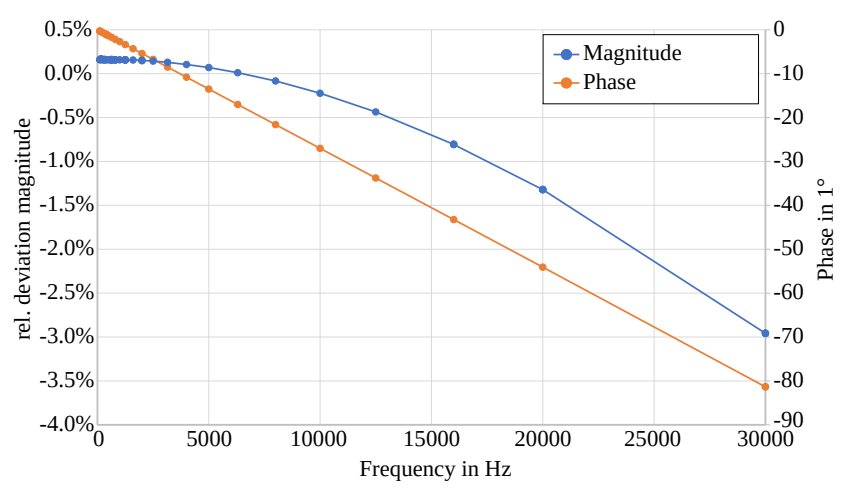

Figure 5: Transfer function of a LDV analog velocity output at $1 \mathrm{~V} /(\mathrm{m} / \mathrm{s})$

diode, simplifying the set-up. It is a classical chickenor-egg dilemma; in our case we had the known PD first to relate an optical signal to an electrical signal.

The measurement uncertainty budget is still under investigation, the absence of mechanical excitation are expected to significantly reduce the uncertainties, leaving the absolute $\mathrm{AC}$ voltage measurement as the main contributor for magnitude uncertainty, while the phase uncertainties are expected to be better than $0.01^{\circ}$ for frequencies up to $100 \mathrm{kHz}$.

\section{ACKNOWLEDGMENT}

The authors like to thank Dr. Siegmund of Polytec for some inspiring walks and talks.

\section{References}

[1] ISO 16063-11:1999 Methods for the calibration of vibration and shock transducers - Part 11: Primary vibration calibration by laser interferometry, ISO, Geneva, Switzerland, 1999

[2] ISO 16063-13:2001 Methods for the calibration of vibration and shock transducers - Part 13: Primary shock calibration using laser interferometry

[3] ISO 16063-41:2011 Methods for the calibration of vibration and shock transducers - Part 41: Calibration of laser vibrometers

[4] U Buehn et al., "Calibration of Laser Vibrometer Standards According to ISO 16063-41", XVIII IMEKO World Congress 2006, Rio de Janeiro, Brazil, September, 2006 https://www.imeko.org/publications/wc2006/PWC-2006-TC22-007u.pdf

[5] Th. Bruns, F. Blume, A. Täubner, "Laser Vibrometer Calibration at High Frequencies using Conventional Calibration Equipment", XIX IMEKO World Congress, September 6-11, 2009, Lisbon, Portugal http://www.imeko2009.it.pt/Papers/FP_495.pdf

[6] F. Blume, A. Täubner, U. Göbel, Th. Bruns, "Primary phase calibration of laser-vibrometers with a single laser source", Metrologia, 2009, Vol. 46, N. 5, https://dx.doi.org/10.1088/0026-1394/46/5/013

[7] M. Winter, H. Füser, M. Bieler, G. Siegmund, C. Rembe, "The problem of calibrating Laser-Doppler Vibrometers at high frequencies", AIP Conference Proceedings 1457, 165 (2012) https://doi.org/10.1063/1.4730555

[8] Th. Bruns, F. Blume, K.Baaske, M. Bieler, H. Volkers "Optoelectronic Phase Delay Measurement for a Modified Michelson Interferometer", Measurement, 2013, Vol.46, N. 5, https://doi.org/10.1016/j.measurement.2012.11.044 